\title{
The Characteristics, Management and Outcome of Patients Receiving Acute Dialysis in Swaziland (Eswatini)
}

\author{
Thandiwe AL Dlamini ${ }^{1 *}$, Tapiwa Chirima², Gode Kinsala ${ }^{3}$, Delisile Simelane ${ }^{1}$, Ntombencane Hlatshwayo ${ }^{1}$, Nkhosikhona A \\ Dlamini $^{1}$, Xolisile Dlamini ${ }^{4}$, Thamie S Kunene ${ }^{5}$, Siphesihle W Dlamini ${ }^{5}$, and Nimrod Matekere ${ }^{3}$
}

${ }^{1}$ Mbabane Government Hospital, Eswatini

${ }^{2}$ Hlathikhulu Government Hospital, Eswatini

${ }^{3}$ Raleigh Fitkin Memorial Hospital, Eswatini

${ }^{4}$ Ministry of Health, Eswatini

${ }^{5}$ University of Swaziland (Eswatini) alumni, Eswatini

*Corresponding author: Thandiwe AL Dlamini, Mbabane Government Hospital, Eswatini, E-mail: taldlamini@gmail.com

Received: 29 Jul, 2019 | Accepted: 28 Aug, 2019 | Published: 03 Sep, 2019

Citation: Dlamini TAL, Chirima T, Kinsala G, Simelane D, Hlatshwayo N, et al. (2019) The Characteristics, Management and Outcome of Patients Receiving Acute Dialysis in Swaziland (Eswatini). Int J Nephrol Kidney Fail 5(2): dx.doi.org/10.16966/2380-5498.180

Copyright: (c) 2019 Dlamini TAL, et al. This is an open-access article distributed under the terms of the Creative Commons Attribution License, which permits unrestricted use, distribution, and reproduction in any medium, provided the original author and source are credited.

\section{Abstract}

Background: Eswatini, a land-locked country in Southern Africa with a population of about 1.4 million, is one of the few countries in Africa where dialysis is available as part of the country's health plan. This is the first study of the pattern of kidney disease amongst patients receiving acute dialysis in the country.

Methods: This was a retrospective folder review of patients who were dialysed at Eswatini's three institutions where dialysis is offered. Patients dialysed acutely from the $1^{\text {st }}$ of June 2015 to the $31^{\text {st }}$ of May 2016 were included. A data collection form was used to collect information on patient demographics (de-identified), co-morbidities, reason for referral for dialysis, place of referral, basic investigations, and outcome in terms of renal recovery, need for chronic dialysis and 30 day mortality. The study was approved by the National Health Research Review Board, the Ethics committee of Eswatini. A waiver of consent was granted as patient information was de-identified. Data from the data collection forms was entered into Microsoft Excel and RStudio software for analysis by the principal investigators.

Preliminary Results: A total number of 219 patients received acute dialysis over the study period. Ages ranged from 9 months to 84 years. Most underwent acute haemodialysis with only 4 undergoing acute peritoneal dialysis. The majority (131) $59.8 \%$ were male. Co-morbidities included HIV in 124 (56.6\%), hypertension in 118 (54.1\%), diabetes in 48 (21.9\%), and urinary tract obstruction in $13(5.9 \%)$. A total of 87 (39.7\%) patients had received Tenofovir Disoproxil Fumarate for treatment of HIV. Of the patients with available data, those achieving partial or full renal recovery within 30 days totaled $42(19.2 \%)$, and 88 (40.2\%) went onto chronic dialysis. There were 31 deaths (out 189 identified folders) (16.4\%) within 30 days.

Conclusions: Dialysis requiring patients in Eswatini include the young and old. The most notable risk factors are hypertension and HIV, as well as recent use of Tenofovir Disoproxil Fumarate. A large proportion of patients present with advanced kidney disease that requires ongoing renal replacement therapy. Improved management of the identified risk factors for acute and chronic kidney disease may reduce the number of patients requiring dialysis in Eswatini.

Keywords: Dialysis; Renal recovery; Mortality

\section{Introduction}

Kidney disease may occur due to various conditions that cause acute or chronic kidney damage. The result is the disturbance of some or all of the functions of the kidneys, including electrolyte, acid-base, and fluid balance. It is a very serious illness that can lead to several complications including death if not treated. Epidemiological studies have clearly demonstrated the association between both acute and chronic kidney disease and increased mortality risk $[1,2]$.
Acute kidney injury is defined by deterioration of kidney function involving either an increase in serum creatinine by more than one and a half times from baseline within 7 days, an increase in serum creatinine by $26.4 \mu \mathrm{mol} / \mathrm{l}$ within a 48 hour period, or a decrease in urine output by at least $0.5 \mathrm{ml} / \mathrm{kg} /$ hour over 6 hours [3]. It is staged according to severity from 1 to 3 [3].

Chronic kidney disease is present if a patient has had kidney abnormalities for at least 3 months, involving either the structure of the kidneys, such as scarring, a urine abnormality such as the presence 
of abnormal amounts of protein, or a decrease in the glomerular filtration rate of the kidneys [4]. It is staged according to severity from 1 to $5[4]$.

Detection of both acute kidney injury and chronic kidney disease in their early stages may provide the opportunity to institute management that can slow progression to a more severe and life threatening stage $[5,6]$. However, patients surviving an acute kidney injury episode have been shown to remain at increased future risk of developing chronic kidney disease, thus contributing to the burden of chronic kidney disease in a population [7].

Although some causes of acute and chronic kidney disease are not preventable such as genetic or autoimmune causes, many potential causes can be avoided by educating the public and health workers. Potentially preventable causes of acute kidney injury described in Africa include dehydration, sepsis and herbal toxins [8,9]. Modifiable causes of chronic kidney disease that are reported in Sub-Saharan Africa include diabetes, hypertension, and HIV [10].

In Eswatini acute and chronic haemodialysis and peritoneal dialysis are offered to patients. Acute peritoneal dialysis is offered to the paediatric age group as well as to patients at the National TB Hospital, treated for drug resistant tuberculosis, who are unable to access the three dialysis centres because of infection control precautions. Haemodialysis is offered at Mbabane Government hospital (the main referral hospital), Raleigh Fitkin Memorial Hospital and Hlathikhulu Government Hospital only.

All three hospitals have laboratories and radiology departments. The Kidney Disease Improving Global Outcomes (KDIGO) group has listed some basic investigations in the management of patients presenting with acute kidney failure [5]. These include laboratory tests (serum creatinine, blood urea nitrogen, and electrolytes), urine analysis (dipstick, microscopy and electrolytes), and abdominal imaging(ultrasonography and computed tomography as indicated) [5].

The treatment of kidney failure with dialysis carries high economic costs, with estimates in South Africa at about ZAR200, 000-00 per patient per year [11].

In view of the personal and national costs of kidney disease, it is vital that measures be taken to sensitise the public and medical staff to this condition, as well as to emphasise the need for its prevention and early detection.

Knowledge of the pattern and outcome of kidney disease in the country can help inform public policy at the national level and improve staff training and public awareness campaigns.

This study was designed to address the gap in the body of knowledge on kidney disease in Eswatini, and to provide useful information for policy makers.

\section{Methods}

\section{Study design}

A descriptive descriptive retrospective design.

\section{Primary aim}

To determine the 30 day mortality rate amongst patients who received acute dialysis from the $1^{\text {st }}$ of June 2015 to the $31^{\text {st }}$ of May 2016.

\section{Secondary aim}

To study the demographics, causes of kidney failure, management and renal outcomes of the population group studied.

\section{Setting and study population}

The study included all patients referred for acute dialysis at each of the country's three facilities that offer dialysis. The population of Eswatini of about 1,4 million people is subdivided into four regions, namely Hhohho with a subpopulation of 270000, Manzini with 319530 , Shiselweni with 217000 and Lubombo with 202000 [12,13]. HIV/AIDS and tuberculosis affect a significant proportion of the population [12]. There is also a documented rise in non-communicable diseases in the country [14].

The three dialysis centres servicing Eswatini are located in three of the country's regions, namely Hhohho (Mbabane Government Hospital, the main Referral Hospital, with dialysis services since 2001), Manzini (Raleigh Fitkin Memorial Hospital with dialysis services since 2011), and Shiselweni (Hlathikhulu Government Hospital with dialysis services since 2013).

\section{Dialysis Protocol}

Acute dialysis was commenced on patients with kidney failure presenting with uraemic features, metabolic acidosis, refractory hyperkalaemia or pulmonary oedema.

\section{Data collection}

The study investigators collected data from the unit registers and patient files and recorded it on the data collection form.

\section{Data analysis}

Information from the data collection forms was entered into Microsoft Excel and RStudio software for analysis by the principal investigators. Due to missing data, only very basic statistical analyses were performed such as mean age and percentage proportions.

\section{Ethical Considerations}

The study was approved by the Eswatini Ethics committee, the National Health Research Review Board. Permission for the study was also sought from the administration boards of the individual hospitals and from the Ministry of Health. A waiver of consent was granted for the study as patient data was de-identified to respect confidentiality.

\section{Results \\ Demographics}

A total of 219 patients received acute dialysis between the $1^{\text {st }}$ of June 2015 and the $31^{\text {st }}$ of May 2016, at the three institutions providing dialysis in Eswatini. Of these, 131 were male (59.8\%) and 88 were female $(40.2 \%)$. The mean age for all the patients was 48.6 years. The mean age of males at 51.6 years was slightly higher than that of females which was 44.8 years. Most patients were treated with haemodialysis with only 4 treated with acute peritoneal dialysis.

\section{Renal outcomes}

Only 189 of the 219 study patients (86.3\%) had data on 30 day mortality, and out of these 189 patients, 31 were recorded as dead within 30 days $(16.4 \%)$. The total going on to chronic dialysis was 88 out of the total of 219 patients $(40.2 \%)$. Among the 118 patients with data for renal recovery, a total of 42 achieved complete or partial renal recovery (35.6\%). A total of 15 out of the 177 patients with data 
(18.5\%) self-discharged and were then lost to follow up within the study period (Table 1).

\section{Comorbid diseases}

The leading comorbidity amongst all the patients was HIV at 57.1\%, followed by Hypertension at $54.1 \%$ (Table 2).

\section{Causative factors and associations leading to the need for acute dialysis}

The commonest pathophysiological factor leading to the need for acute dialysis was pre-renal at $49.7 \%$. This was followed by the finding that $42.2 \%$ of the patients with non-missing data were on Tenofovir Disoproxil Fumarate (TDF) at the time of detection of their kidney failure (Table 3). There were 13 out of $158(8.2 \%)$ patients with underlying urinary tract obstruction and 24 out of 156 (15.4\%) had a recent history of ingesting herbal medication.

\section{Patients receiving the basic set of investigations}

Only $102(46.6 \%)$ of the patients studied received the complete recommended basic set of investigations for patients presenting with kidney failure (Figure 1).

\section{Discussion}

This is the first study to attempt to describe the population of people receiving acute dialysis in Eswatini. As it was a retrospective study, there was a lot of incomplete data, which hampered the ability to analyse the data for associations between certain variables and patient outcomes. However, the basic data that was obtained sheds some light on the population receiving acute dialysis in Eswatini.

The finding that most of the patients receiving dialysis were male was consistent with published data showing that more males receive dialysis than females [15]. This is despite the fact that chronic kidney disease is reported more in females than in males [16].

The overall 30 day mortality rate of $16.4 \%$ amongst patients with non-missing data included both patients with acute kidney injury and those who had end stage renal disease. A Cape Town study looking at patients with all stages of acute kidney injury, found a 30 day mortality of $37.6 \%$ amongst those who were dialysed [17].

A big concern from the data is the large proportion of patients who went on to require chronic dialysis. This suggests earlier stages of their chronic kidney disease was missed. Standard practice is that patients with chronic kidney disease be detected early and followed up at clinics where steps to slow or even prevent progression to end stage renal disease can be instituted [6].

The two leading comorbidities were HIV and hypertension, with diabetes mellitus only reported in $22.4 \%$ of the patients with available data. The high number of patients with HIV reflects the current high prevalence of HIV in Eswatini [18]. The significant proportion of patients with hypertension is supported by data from the country's STEPS Non-communicable Risk Factor Survey conducted in 2014 in which untreated hypertension was detected in $78.9 \%$ of the adults studied [19].

Unlike in more developed parts of the world where diabetes mellitus is reported to be a leading cause of chronic kidney disease, together with hypertension, it ranked only third amongst the patients in our data [20]. This may be partly explained by missed diagnosis, due to reduced insulin clearance and gluconeogenesis in the chronically diseased kidneys, leading to deceptively normal blood glucose levels in some patients with diabetic kidney disease [21]. In this study, there
Table 1: A total of 15 out of the 177 patients with data (18.5\%) selfdischarged and were then lost to follow up within the study period.

\begin{tabular}{|l|l|l|l|}
\hline Renal outcome & \multicolumn{1}{|c|}{$\begin{array}{c}\text { Total } \\
\text { identified }\end{array}$} & $\begin{array}{c}\text { Total out of all } \\
\text { uncensored } \\
\text { patients }\end{array}$ & $\begin{array}{c}\text { Total out of all study } \\
\text { patients including } \\
\text { censored patients }\end{array}$ \\
\hline Relapses & 2 & $2 / 166(1.2 \%)$ & $2 / 219(0.9 \%)$ \\
\hline Self-discharged & 15 & $15 / 177(8.5 \%)$ & $15 / 219(6.8 \%)$ \\
\hline $\begin{array}{l}\text { Overall Dead in } \\
\text { 30 days }\end{array}$ & 31 & $31 / 189(16.4 \%)$ & $31 / 219(14.2 \%)$ \\
\hline Transfer outs & 33 & $33 / 177(18.6 \%)$ & $33 / 219(15.1 \%)$ \\
\hline Renal recovery & 42 & $42 / 118(35.6 \%)$ & $42 / 219(19.2 \%)$ \\
\hline Chronic dialysis & 88 & $88 / 219(40.2 \%)$ & $88 / 219(40.2 \%)$ \\
\hline
\end{tabular}

Table 2: The leading comorbidity amongst all the patients was HIV at $57.1 \%$, followed by Hypertension at $54.1 \%$.

\begin{tabular}{|l|c|c|c|}
\hline \multicolumn{1}{|c|}{ Condition } & $\begin{array}{c}\text { Total } \\
\text { identified }\end{array}$ & $\begin{array}{c}\text { Total out of all } \\
\text { uncensored } \\
\text { patients }\end{array}$ & $\begin{array}{c}\text { Total out of all study } \\
\text { patients including } \\
\text { censored patients }\end{array}$ \\
\hline $\begin{array}{l}\text { Diabetes } \\
\text { Mellitus }\end{array}$ & 48 & $48 / 214(22.4 \%)$ & $115 / 214(53.7 \%)$ \\
\hline Hypertension & 118 & $118 / 218(54.1 \%)$ & $118 / 219(54.1 \%)$ \\
\hline HIV & 124 & $124 / 217(57.1 \%)$ & $124 / 219(56.6 \%)$ \\
\hline HIV on ART & 115 & $115 / 214(53.7 \%)$ & $115 / 219(52.5 \%)$ \\
\hline
\end{tabular}

was no provision to look for other diabetes related target organ damage such as diabetic retinopathy. Reports on the leading causes of chronic kidney disease in sub-Saharan Africa, also found that diabetes was not the most common cause [22].

Regarding the causative factors and associations for the need for acute dialysis, pre-renal causes were common. From the data collection sheets, only 44 patients had a clear indication of the cause of acute kidney injury, such as gastroenteritis and septic shock. There were no recorded cases of Haemolytic Uraemic Syndrome. Data from other regions of Africa also report a predominance of pre-renal causes of acute kidney injury such as diarrhoeal diseases [23].

The use of TDF was found in a large proportion of the patients requiring acute dialysis. This study was not designed to detect whether or not TDF was the cause of kidney failure in the patients, and it can therefore only be said to be an association at this stage. However the finding of kidney failure necessitated the removal of this valuable antiretroviral drug from patients's treatment armamentarium. TDF has been shown to cause both acute and chronic kidney disease in studies [24]. Guidelines exist to identify patients who are most at risk of TDF associated kidney disease [25]. A recommendation from the study is that these guidelines be emphasised at the time of initiation of antiretroviral therapy. The adoption of guidelines to monitor for TDF associated proximal renal tubular injury, using urine dipsticks, could assist with the early detection and management of affected patients in the country [26].

A recent history of ingesting herbal medication was detected in $15.4 \%$ of the patients, which is not an insignificant figure. Many indigenous plants have proven health benefits, however, analysis of these plants to elicit their therapeutic and toxic dose, in order to facilitate safe prescription is essential.

The association of Non-Steroidal Anti-Inflammatory Drug (NSAID) use was detected in $7.1 \%$ of the patients. These are drugs that 
can be easily accessed over the counter in the country. Although their prescription in the short-term may well be necessary, their use needs to be held off in the presence of reduced blood pressure and worsening kidney failure as they may cause or exacerbate kidney failure [27]. The implementation of the Medicine's control Bill in Eswatini should help in preventing people from self-medicating with these drugs. Education of Health workers is key in ensuring safe prescription of non-steroidal anti-inflammatory drugs.

The finding of kidney disease arising from urological tract obstruction underlines the need for increased Urological services in the country. Often early detection and treatment of urinary tract obstruction facilitates treatment that can circumvent the need for dialysis [28].

Only $46.6 \%$ of the patients had the full set of recommended basic investigations as outlined by KDIGO for patients presenting with

Table 3: The finding that $42.2 \%$ of the patients with non-missing data were on Tenofovir Disoproxil Fumarate (TDF) at the time of detection of their kidney failure.

\begin{tabular}{|l|c|c|c|}
\hline AKI cause/association & $\begin{array}{c}\text { Total } \\
\text { identified }\end{array}$ & $\begin{array}{c}\text { Total out of all } \\
\text { uncensored } \\
\text { patients }\end{array}$ & $\begin{array}{c}\text { Total out of all } \\
\text { study patients } \\
\text { including } \\
\text { censored patients }\end{array}$ \\
\hline Aminoglycosides & 1 & $1 / 155(1.8 \%)$ & $1 / 219(0.5 \%)$ \\
\hline Malaria & 1 & $1 / 219(0.5 \%)$ & $1 / 219(0.5 \%)$ \\
\hline Pregnancy & 4 & $4 / 219(1.8 \%)$ & $4 / 219(1.8 \%)$ \\
\hline $\begin{array}{l}\text { Non steroidal anti- } \\
\text { inflammatory drugs }\end{array}$ & 11 & $11 / 155(7.1 \%)$ & $11 / 219(5 \%)$ \\
\hline $\begin{array}{l}\text { Urinary tract } \\
\text { obstruction }\end{array}$ & 13 & $13 / 158(8.2 \%)$ & $13 / 219(5.9 \%)$ \\
\hline Herbal remedy use & 24 & $24 / 156(15.4 \%)$ & $24 / 219(11 \%)$ \\
\hline $\begin{array}{l}\text { Pre-renal causes } \\
\text { (diarrhoeal illness/ } \\
\text { gastroenteritis=35; } \\
\text { septic shock=7; } \\
\text { haemorrhage=1; } \\
\text { heart failure=1; } \\
\text { unspecified=36) }\end{array}$ & 80 & $80 / 161(49.7 \%)$ & $80 / 219(36.5 \%)$ \\
\hline TDF use & 87 & $87 / 206(42.2 \%)$ & $87 / 219(39.7 \%)$ \\
\hline
\end{tabular}

Patients with investigations out of the total of 219 Study patients

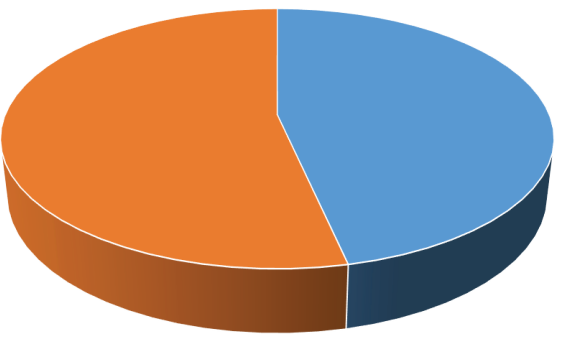

" Complete investigations 102/219 (46.6\%) " Incomplete investigations 117/219 (53.4\%)

Figure 1: Patients receiving the basic set on investigations.

Only $102(46.6 \%)$ of the patients studied received the complete recommended basic set of investigations for patients presenting with kidney failure. kidney failure. Some of these are simple to do, such as urine dipsticks. Basic ultrasonography of the kidneys, ureters and bladder can be taught to medical officers.

\section{Study limitations}

The accuracy of our data results was limited by the incompleteness of patient records in the three institutions. This lack of complete data necessitated the censoring and omission of patients with missing information in the calculations of many of the variables examined in the study. This was done in order to reduce bias in reporting. The only data that was detected from the total number of 219 patients was that for malaria, pregnancy, those with a complete set of investigations and those going on to chronic dialysis.

Due to incomplete data, it was not possible to accurately compare data per institution. Furthermore, it was not possible to ascertain an association between 30 day mortality and gender, HIV status or age.

Although hypertension was one of the leading co-morbidities, the investigations performed were not adequate enough to identify those with hypertension secondary to underlying renal or renovascular disease.

Another limitation is that none of the patients had a kidney biopsy. This is due to resource limitations such as the lack of biopsy needles and fully equipped pathology services. It is hoped that with the advancement of kidney service delivery in the country, kidney biopsies will be performed at Mbabane Government Hospital, the main referral hospital.

\section{Conclusion}

A large proportion of those requiring acute dialysis during the study period were patients with missed chronic kidney disease, who went onto require chronic dialysis.

The leading co-morbidities amongst the patients studied included a communicable disease-HIV and a potentially modifiable noncommunicable disease -hypertension.

Many causes of dialysis receiving kidney failure were found to be conditions that can be targeted by population wide education (such as early rehydration for people with diarrhoea and vomiting, avoiding toxic herbal remedies and avoiding self-medicating with non-steroidal anti-inflammatory drugs), as well as screening for conditions that can lead to urinary tract obstruction-such as prostate cancer and cancer of the cervix.

The finding of TDF use in $42.2 \%$ of patients dialysed for kidney failure indicates the need for greater adherence to the provided screening tools to detect patients at high risk for TDF induced kidney disease at the time of anti-retroviral therapy initiation. In addition, point of care tests such as a urine dipstick and bedside serum creatinine analysis to enable early detection of TDF induced acute kidney injury are highly recommended.

Although limited by the nature of a prospective observational study, some important insights in to the characteristics of patients requiring acute dialysis in Eswatini have been gained by the study. It is anticipated that the information gathered can be employed by relevant stakeholders to reduce the burden of kidney disease in the country. Follow-up studies of higher quality design would be useful.

\section{Acknowledgments}

This study would not have been possible without Dr. Nimrod Matekere, who founded the Renal Replacement Therapy Programme in Eswatini. 
The study was self-funded by the investigators at a total sum amounting to an equivalent of $\$ 10$ (ten United States Dollars).

\section{Conflict of Interest Statement}

The Principal Investigator has a Consultancy contract with Fresenius Medical Care, which has a Public Partnership Agreement with the Government of Eswatini at one of the study centres (Mbabane Government Hospital).

\section{References}

1. Waikar SS, Liu KD, Chertow GM (2008) Diagnosis, Epidemiology and Outcomes of Acute Kidney Injury. Clin J Am Soc Nephrol 3: 844-861.

2. Tonelli M, Wiebe N, Culleton B, House A, Rabbat C, et al. (2016) Chronic kidney disease and mortality risk: a systematic review. J Am Soc Nephrol 17: 2034-2047.

3. Bellomo R, Ronco C, Kellum JA, Mehta RL, Palevsky P, et al. (2004) Acute renal failure-definition, outcome measures, animal models, fluid therapy and information technology needs: the Second International Consensus Conference of the Acute Dialysis Quality Initiative (ADQI) Group. Crit Care 8: R204-R212.

4. Levey AS, Eckhardt KU, Tsukamoto Y, Levin A, Coresh J, et al. (2005) Definition and classification of chronic kidney disease: a position statement from Kidney Disease: Improving Global Outcomes (KDIGO). Kidney Int Suppl 67: 2089-2100.

5. KDIGO (2012) KDIGO Clinical Practice Guideline for Acute Kidney Injury. Kidney Int Suppl 2: 28.

6. KDIGO (2013) KDIGO 2012 Clinical Practice Guideline for the Evaluation and Management of Chronic Kidney Disease Kidney. Kid Int Suppl 3: 1-150.

7. Venkatachalam MA, Griffin KA, Lan R, Geng H, Saikumar P, et al. (2010) Acute kidney injury: A springboard for progression in chronic kidney disease. Am J Physiol Renal Physiol 298: F1078-F1094.

8. Kaballo BG, Khogali MS, Khalifa EH, Khaiii EA, Ei-Hassan AM, et al. (2007) Patterns of "severe acute renal failure" in a referral center in Sudan: excluding intensive care and major surgery patients. Saudi J Kidney Dis Transpl 18: 220-225.

9. Cerda J, Lameire N, Eggers P, Pannu N, Uchino S, et al. (2008) Epidemiology of acute kidney injury. Clin J Am Soc Nephrol 3: 881886.

10. Stanifer JW, Jing B, Tolan S, Helmke N, Mukerjee R, et al. (2014) The epidemiology of chronic kidney disease in Sub-Saharan Africa: a systematic review and meta-analysis. Lancet Glob Health 2: e174-e181.

11. Moosa MR, Meyers AM, Gottlich E, Naicker S (2016) An effective approach to chronic kidney disease in South Africa. S Afr Med J 106: 156-159.
12. Wikipedia (2016) Swaziland.

13. Central Intelligence Agency (2005) The World Factbook. Eswatini.

14. Analytical summary Non-communicable diseases and conditions. World Health Organisation (WHO), African Health Observatory, Africa.

15. Hecking M, Bieber BA, Ethier J, Kautzky-Willer A, Sunder-Plassmann $G$, et al. (2014) Sex-specific differences in haemodialysis prevalence and practices and the male-to-female mortality rate: The Dialysis Outcomes and Practice Pattern Study (DOPPS). PLoS Med 11: e1001750.

16. Editorial (2018) Women's health and Kidney disease. Nat Rev Nephrol 14: 139.

17. Dlamini TAL, Heering PJ, Chivese T, Rayner B (2017) A prospective study of the demographics, management and outcome of patients with acute kidney injury in Cape Town, South Africa. PLoS One 12: e0117460.

18. Justman J, Reed JB, Bicego G, Donnell D, Li K, et al. (2017) Swaziland HIV Incidence Measurement Survey (SHIMS): a prospective national cohort study. Lancet HIV 4: e88-e92.

19. Fact Sheet (2014) Swaziland STEPS Survey 2014.

20. Webster AC, Nagler EV, Morton RL, Masson P (2017) Chronic kidney disease. Lancet 389: 1238-1252.

21. Alsahli M, Gerich JE (2014) Hypoglycaemia, chronic kidney disease, and diabetes mellitus. Mayo Clin Proc 89: 1564-1571.

22. Arogundade FA, Barsoum RS (2008) CKD prevention in Sub-Saharan Africa: A call for Governmental, Non-Governmental, and Community support. Am J Kidney Dis 51: 515-523.

23. Naicker S, Aboud O, Gharbi MB (2008) Epidemiology of acute kidney injury in Africa. Semin Nephrol 28: 348-353.

24. Fernandez-Fernandez B, Montoya-Ferrer A, Sanz AB, Sanchez-Niño MD, Izquierdo MC, et al. (2011) Tenofovir Nephrotoxicity: 2011 Update. AIDS Res Treat 2011: 354908.

25. Górriz JL, Gutiérrez F, Trullas JC, Arazo P, Arribas JR, et al. (2014) Consensus document on the management of renal disease in HIVinfected patients. Nefrologia 34: 1-18.

26. Boswell MT, Rossouw TM (2017) Approach to acute kidney injury in HIV infected patients in South Africa. South Afr J HIV Med 18: 714.

27. Whelton A, Hamilton CW (1991) Nonsteroidal anti-inflammatory drugs: effects on kidney function. J Clin Pharmacol 31: 588-598.

28. Klahr S (2000) Obstructive nephropathy. Intern Med 39: 355-361. 